\title{
AVANCES EN EL ESTUDIO DE ÁREAS DE ACTIVIDAD DOMÉSTICA EN EL SITIO DE PUEBLO VIEJO DE TUCUTE (PUNA DE JUJUY). ANÁLISIS DE LA CERÁMICA DEL RECINTO R3.
}

\author{
Diego Martín Basso ${ }^{(1)}$, Noelia Lizarraga( ${ }^{(1)}$ María Tejerina $^{(1)}$ y María \\ Zaburlín ${ }^{(2)}$ \\ (1) FHyCS-UNJu \\ (2) Docente Carrera de Antropología. CREA - FHyCS-UNJu \\ Presentado el: 10/03/2009 - Aceptado 18/06/2009
}

\section{Resumen}

Se desarrolla un análisis de áreas de actividad en el recinto 3 (R-3) del sitio Pueblo Viejo de Tucute (Tu-1). Se priorizaron los datos del material cerámico proveniente del piso de ocupación y su distribución espacial. En este trabajo también se incluyen datos parciales sobre las distribuciones de otros materiales arqueológicos registrados en el recinto (restos de fauna, elementos líticos de diversas clases, restos macro vegetales) aunque es necesario mencionar que los mismos están en proceso de análisis.

Por último se realiza una comparación con otra vivienda circular excavada en el sitio.

Palabras Clave: Áreas de actividad, cerámica, vivienda.

\begin{abstract}
There develops an analysis of areas of activity in the enclosure $3(R-3)$ of the site Pueblo Viejo de Tucute (Tu-1). There were prioritized the information of the ceramic material from the floor of occupation and his spatial distribution. In this work also partial information is included on the distributions of other archaeological materials registered in the enclosure (remains of fauna, elements lithics of diverse classes, remains macro vegetable) though it is necessary to mention that the same ones are in process of analysis.

Finally a comparison is realized by another circular housing excavated in the site.
\end{abstract}

Key Words: Activity areas, pottery, circular dwelling.

\section{Introducción}

Pueblo Viejo de Tucute (en adelante PV de Tucute) se ubica en la quebrada homónima al suroeste del poblado de Casabindo en la Puna de Jujuy $\left(23^{\circ} 01^{\prime} 68\right.$ de latitud sur y $66^{\circ} 04^{\prime} 64,7$ de longitud oeste: a 3.757 m.s.n.m). Se trata de un poblado conglomerado donde se registran más de 500 recintos con planta circular y cerca de una decena con planta rectangular. El asentamiento se distribuye sobre dos elevaciones, separadas por un arroyo, que fueron denominadas Loma Baja y Loma Alta (Albeck y Zaburlín 2007). 


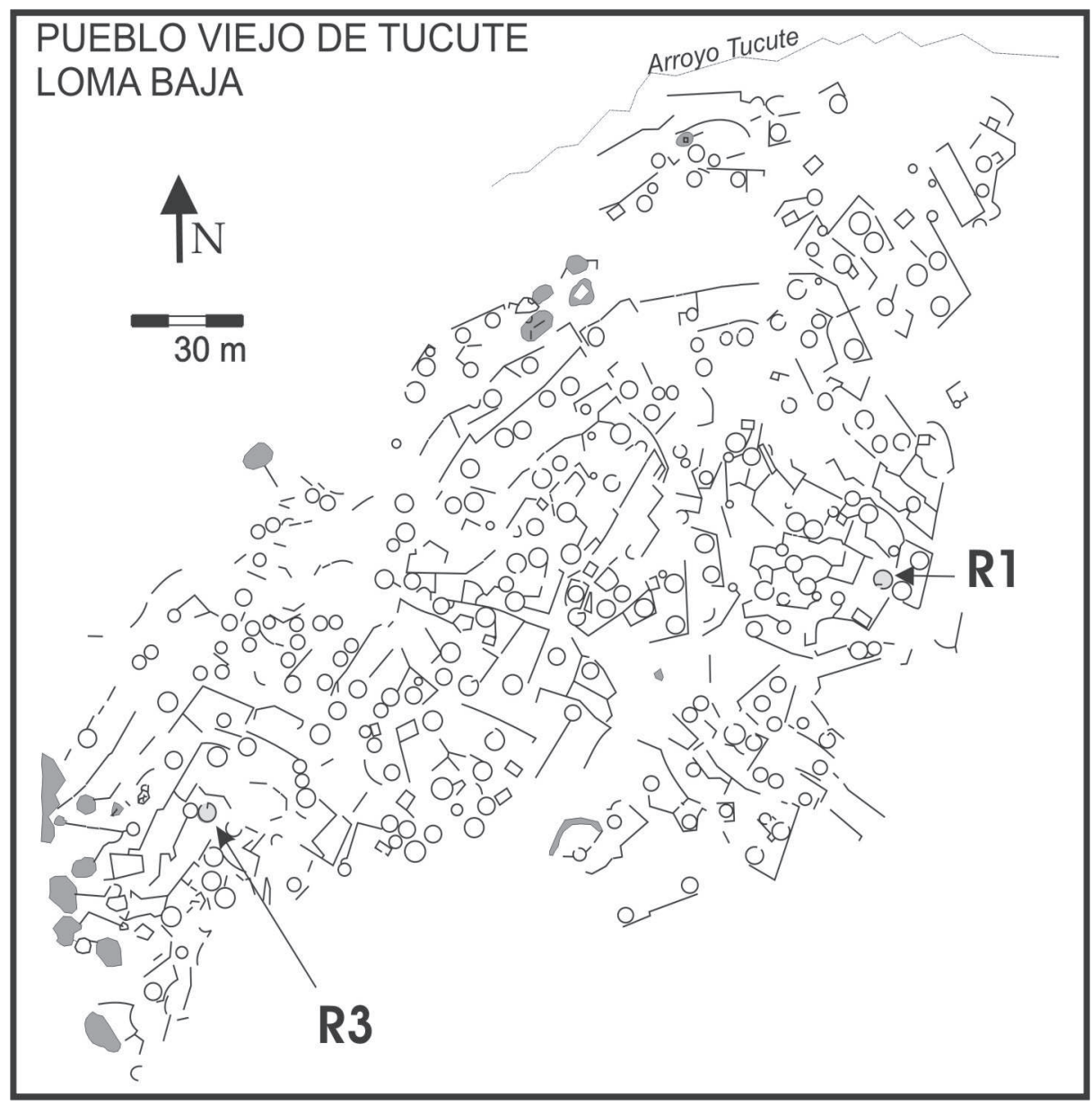

Figura 1: Pueblo Viejo de Tucute. Loma Baja.

El recinto R3 corresponde a una vivienda de planta circular ubicada en el sector suroeste de la Loma Baja (Figura 1).Un fechado obtenido en este recinto ubica el inicio de su ocupación en el siglo XI (Albeck y Zaburlín 2008:166).

Hasta el momento se ha planteado que los recintos circulares individuales son estructuras multifuncionales principalmente utilizadas como viviendas. Sin embargo, todavía no se puede definir el equivalente arquitectónico del grupo corresidencial mínimo, ya que puede implicar cada recinto individual o un conjunto de recintos.

Al tratarse de recintos circulares no asociados los cuales no evidencian modificaciones en la estructura, ni división del espacio interior mediante muros o estructuras adyacentes adosadas en los lados no se puede hacer un análisis de uso del espacio mediante los datos arquitectónicos, por eso se recurre al estudio de la distribución espacial de los materiales, cuya ubicación esta medianamente condicionada por algunos elementos semifijos.

\section{Antecedentes}

El Área de Pueblo Viejo de Tucute fue visitada por Uhle, Gerling y von Rosen entre fines del siglo XIX y principios del XX, los materiales exhumados en estas campañas provienen 
principalmente de Chullpas o cuevas tapiadas. En 1938 Casanova retomó los trabajos en el sitio y lo denominó Sorcuyo, hasta el momento se desconoce si en esa ocasión se excavaron recintos habitacionales (para una revisión más detallada ver Albeck 2010 e. p.). Desde 1980 está siendo trabajado por Maria Ester Albeck con distintos proyectos de investigación que fueron apuntando a estudiar diversos aspectos del sitio, tanto a nivel macro como semimicro y micro.

En Pueblo Viejo de Tucute las construcciones se asientan sobre faldeos con fuertes pendientes niveladas por muros de contención, sobre estos espacios se ubican conjuntos de recintos de planta circular. Se trata de unidades aisladas sin patios formales, se registraron 258 recintos circulares en la Loma Baja y 209 en la Loma Alta.

Las estructuras circulares presentan una arquitectura bastante homogénea. El espacio interior está delimitado por un solo muro levantado con rocas cortadas en forma prismática, con las hiladas dispuestas como en una pared de mampostería. Es recurrente la presencia de un deflector de aire dispuesto de forma oblicua junto al acceso, este puede ser monolítico o formado por una pequeña pared. Casi al centro de los recintos se ubica un fogón construido con barro y piedras. Hasta el momento se desconoce la técnica de techado.

Las diferencias entre los recintos consisten principalmente en las dimensiones, orientación del acceso y en la materia prima con la que fueron construidos, que corresponden a variedades de rocas locales (para más detalles ver Albeck et al 1998; Albeck y Zaburlín 2007).

Como antecedente específico se cuenta con un análisis de áreas de actividad para otro recinto circular, denominado R1, también ubicado en la Loma Baja de P.V. de Tucute (Albeck 1997, 1999; Albeck et al 1995). En el recinto R1 se identificaron dos niveles en el piso de ocupación. El nivel superior correspondía al evento de abandono, de donde se remontaron 10 vasijas completas entre la basura de facto (Albeck et al. 1995:206). El nivel inferior, en cambio, estaba conformado por el palimpsesto generado durante la ocupación del recinto.

La reconstrucción de áreas de actividad considerando el nivel superior del piso de ocupación del R1 indica que allí se realizaron actividades de procesamiento de alimentos, algunas etapas de manufactura cerámica y lítica y también eventos de inhumación (Albeck 1997,1999).

Las vasijas remontadas, correspondientes al momento de abandono, permitieron identificar áreas de almacenaje junto a los muros y actividades de preparación y consumo de alimentos en el área cercana al fogón (Albeck et.al. 1995: 219-220). En este artículo los autores plantearon el problema de la definición del tipo de abandono y las expectativas de registrar basura de facto (sensu Schiffer 1979) en contextos domésticos. Estos contextos, como el del $\mathrm{R} 1$, con abundante basura de facto ¿implican un abandono repentino con los elementos dejados en su lugar de uso? o ¿acaso se trata de un abandono temporal donde las piezas fueron guardadas previendo un retorno? (Albeck et al. 1995: 219-220).

El contraste entre ambas interpretaciones es importante, ya que si se pretende comparar unidades domésticas, la presencia de una cantidad considerable de piezas para almacenaje puede implicar diferencias de acceso o control de ciertos recursos almacenados. En cambio si las piezas estaban guardadas no implicaría directamente una diferenciación social (Menacho 2000). 


\section{Herramientas teóricas}

La arquitectura participa en un sistema social de comunicación no verbal, donde el espacio físico comunica aspectos de la identidad social e individual entre los integrantes de la unidad doméstica y hacia el exterior de la misma (Blanton 1994:8). En el interior de la vivienda, la arquitectura, organización y uso de los espacios conforman un medio de comunicación entre sus propios ocupantes. Es el marco material que estructura la vida cotidiana y los rituales domésticos, abarcando la reproducción social de la unidad doméstica (Blanton 1994:10-80-81 citando a Rapoport 1969, Bourdieu 1973 y otros).

Se ha planteado que en Pueblo Viejo de Tucute la modalidad de organización del espacio habitado y las condiciones topográficas generaron en las áreas abiertas, en mayor o menor grado, una alta exposición visual y acústica entre los antiguos habitantes del pueblo. Únicamente el interior de las viviendas puede considerarse como un espacio de acceso restringido, reservado e íntimo (Albeck y Zaburlín 2007:176).

De ahí la importancia que otorgamos a los estudios de áreas de actividad en este sitio, ya que mediante la comparación de las actividades realizadas en el interior de los recintos esperamos avanzar en el análisis de:

- Diferencias/similitudes entre las viviendas, lo que permitiría identificar diferencias en la población ya sea jerárquicas o según el grado de especialización en la producción. - Diferencias/similitudes en actividades o en la estructuración del espacio en el interior de las viviendas, lo cual nos permitiría analizar las pautas compartidas entre las distintas unidades de vivienda.

El concepto de áreas de actividad en arqueología se refiere principalmente a la distribución de ciertos materiales arqueológicos y su vinculación con las acciones de la gente en el pasado, como lo son las actividades domésticas. Sin embargo se debe considerar que las actividades humanas tienen una organización y una distribución espacial compleja, Rapoport (1990) plantea que no se deben buscar actividades singulares si no más bien considerar sistemas de actividades (activity sistems), los cuales no están organizados en una estructura particular, sino que implican el uso de una variedad de espacios internos y externos, asentamientos, la región, etc.: "activity systems take place in systems of settings" (Rapoport 1990:12).

Este autor también menciona que es útil identificar los tipos de elementos que conforman el contexto de estudio, entre ellos se encuentran los elementos fijos, semi fijos y no- fijos, los cuales brindan un panorama acerca de qué actividades se pueden estar desarrollando en determinadas áreas (Rapoport 1990:13).

Para trabajar sobre áreas de actividad en espacios domésticos se debe considerar que los depósitos de piso son palimpsestos conformados por las actividades productivas, de limpieza y sus desechos, que a su vez fueron afectados con distinta intensidad por procesos naturales (Wandsnider 1996:334). Al mismo tiempo se debe tomar en cuenta que la basura primaria es un buen indicador de las acciones que desarrollaba la gente, brindando indicios del tiempo de uso de la vivienda, por otro lado su distribución no es el resultado intencional de sus habitantes, como sí puede ser la basura de facto (Schiffer 1979:85).

El área excavada con la que contamos está restringida a un recinto, donde se pretenden reconocer algunas actividades a través del análisis de distribución de los materiales, 
principalmente la cerámica, y en lo posible vincularlas con el uso del espacio dentro de la vivienda.

A continuación se exponen los resultados de los estudios sobre áreas de actividad en el R3 de Pueblo Viejo de Tucute. En la primera parte se desarrolla la metodología y en la segunda sección se describe la excavación y características arquitectónicas del recinto R3. Por último se presentan los resultados en cuanto a procesos de formación y del análisis del material cerámico proveniente del piso de ocupación.

\section{Metodología}

El análisis del material se realizó en tres etapas: 1) identificación de procesos de formación; 2) análisis morfológico funcional de las piezas y fragmentos cerámicos y 3) análisis de distribución de materiales en el recinto.

El primer paso consistió en identificar la ocurrencia de procesos de formación postdepositacionales, para prevenir caer en una "premisa Pompeya" (Binford 1981; Schiffer 1988). Además de analizar los informes de campo, se realizaron remontajes con el material cerámico y luego se confeccionaron gráficos con las procedencias de los fragmentos remontados, de manera tal que se pudiese verificar su origen estratigráfico y espacial. Esta metodología fue propuesta por Nelson (1985) para solucionar problemas estratigráficos y también fue aplicada para el R1 (Albeck et al. 1995).

En la etapa de remontaje de la cerámica del R3 se siguieron las propuestas de Bollong (1994), quien plantea una serie de estados presentes en la variedad de piezas fragmentadas y tiestos sueltos que pueden registrarse en una excavación. Este autor sugiere como premisa que en el registro arqueológico "cada tiesto proviene de una vasija completa, que esta pudo o no haber estado presente en el sitio y que todos los fragmentos de la vasija pueden no haber sido desechados en el mismo" (Bollong 1994:17-18). En base a esto propone seis estados de remontaje:

El estado 1 corresponde a los remontajes entre tiestos; los estadios 2 y 3 incluyen fragmentos que no remontan pero por características morfológicas macroscópicas se puede demostrar que pertenecen a una misma región del cuerpo o a una misma vasija. Estos tres primeros estados se consideran de asociación segura.

El estado 4 comprende fragmentos que no remontan pero sus atributos morfológicos macroscópicos permiten establecer asociaciones probables. El estado 5 corresponde a los fragmentos únicos. En base a la morfología macroscópica se puede demostrar que el tiesto es único en el conjunto. Puede representar cerámica introducida en el sitio como un fragmento singular o si la excavación es incompleta puede estar asociado con otros fragmentos de vasijas no recuperadas. La naturaleza distintiva de estos tiestos permite, con precauciones, la designación de vasija.

Por último, el estado 6 incluye a los tiestos huérfanos que son aquellos fragmentos a los que no se les puede reconocer de qué parte de la pieza provienen ni el tipo de vasija que representan. 
En una misma pieza remontada se pueden registrar los tres primeros estados, estos son los que deben utilizarse para el análisis de estratigrafías y de distribución espacial. El material incluido en el estado 4 puede ser empleado para un agrupamiento generalizado y tentativo del material, pero se deben tener precauciones si se pretende utilizar la procedencia de estos fragmentos para un análisis estratigráfico (Bollong 1994:17).

Los fragmentos aislados que se incluyen en los estados 5 y 6 no son operativos para un análisis estratigráfico. Sin embargo, la comparación entre los porcentajes de fragmentos únicos y los remontajes pueden proporcionar información sobre la función del depósito sedimentario del cual provienen. Por ejemplo se espera que en un basurero se registren mayores proporciones y variedades de fragmentos únicos que en contextos habitacionales (Bollong 1994:17).

El segundo paso consistió en clasificar los materiales cerámicos en base a atributos morfofuncionales. Para la descripción morfológica del material cerámico se tomaron los criterios de Sheppard (1965) y para el análisis funcional se siguieron las recomendaciones de Menacho (2007:17), considerando atributos morfo-funcionales y huellas de uso. Esta autora, a partir de estudios etnoarqueológicos, contempla las propiedades formales que rigen la función de las vasijas:

-la receptividad; consiste en la relación entre el diámetro máximo y el diámetro de la abertura, esta propiedad tiene implicancias en la manipulación y acceso al contenido en las vasijas (Menacho 2007:17 y 23). De esta forma se diferenciaron las vasijas de boca ancha de las vasijas cerradas con cuello.

-el tamaño y volumen en litros es una propiedad relacionada principalmente con la transportabilidad de las piezas. El límite para trasladar contenidos ha sido establecido para vasijas que no superan los 15 litros (Menacho 2007:17 y 23).

-La presencia de atributos morfológicos con funciones específicas como el tamaño, la posición de las asas y otras.

Se registraron también huellas de uso a nivel macroscópico, considerando su intensidad y ubicación en la superficie de la pieza.

Una vez clasificados los fragmentos y las vasijas según los tipos de piezas reconocidos para la región, se procedió a calcular el número mínimo de individuos. Para esto se tuvieron en cuenta los fragmentos con atributos morfológicos, como por ejemplo los bordes y las bases, siempre y cuando se pudiera determinar que provenían de vasijas diferentes. Así por ejemplo, si en los pucos se contaron 14 bordes provenientes de diferentes piezas, mientras que se contabilizaron 4 bases también pertenecientes a diferentes vasijas, se puede suponer que las cuatro bases podrían corresponderse con algunos de los bordes, por lo tanto el número mínimo serían los 14 individuos representados por los bordes. Al tener un número mayor de ciertos elementos sobre otros se considera que estos contienen a los de número inferior. A esos se les suma, además, aquellos fragmentos de cuerpo que presentan características especiales que los distinguen del conjunto.

Para obtener el número máximo de vasijas representadas se contabilizaron todos los fragmentos que pertenecían a cada tipo morfológico de vasija identificada. Las medidas de número mínimo y máximo deben ser usadas conjuntamente para tener en cuenta el rango probable de piezas representadas en el piso de ocupación del recinto excavado. 
En el tercer paso se realizó un análisis de la distribución de materiales en el espacio del recinto. Con este fin todos los materiales cerámicos registrados en la excavación fueron clasificados en relación a las etapas del ciclo de vida y el tipo de desecho que representaban (Schiffer 1979:85). Se confeccionaron mapas con las distribuciones de fragmentos para cada una de las piezas remontadas y uno con todos los fragmentos afectados por exposición al fuego.

Además se dibujaron plantas discriminando los materiales exhumados como cerámica, óseo, lítico y luego se identificaron los tipos de desecho que conformaban: basura primaria o desecho de facto. En base a estos planos se analizaron las relaciones espaciales entre los materiales y los rasgos arquitectónicos, con el fin de identificar posibles áreas de actividad dentro de la vivienda.

\section{El recinto R3}

Se eligió excavar el recinto R3 porque en superficie aparentaba tener una estructura menos conservada que el R1, sólo se podía observar parte de un muro con forma circular de $20 \mathrm{~cm}$ de altura en el sector sur, en tanto en el flanco oeste la pared se encontraba derrumbada y cubierta por sedimento eólico, además el material con el que fue construido pertenece a una variedad de roca que se desintegra con facilidad. Estos indicadores en un principio se consideraron como probables señales de diferencia cronológica con el R1.

Las excavaciones se llevaron a cabo en campañas realizadas en los años 2000, 2004 y 2005. En la primera se realizó un sondeo inicial de $1 \times 1 \mathrm{~m}$ en el sector oriental del recinto con el objeto de reconocer la estratigrafía. Al inicio de las últimas campañas se constató que el sedimento había sido removido por huaqueros, pero el daño no fue importante debido a que sólo afectaron sectores ya registrados.

Se plantearon 22 cuadrículas de $1 \times 1$ m que se excavaron mediante la técnica de decapage; se utilizó malla de $0,2 \mathrm{~cm}$. para zaranda. En la excavación se reconocieron cuatro estratos:

- Estrato I, corresponde a los sedimentos superficiales: aquí se encontró gran cantidad de piedras pertenecientes al derrumbe del muro, escaso material cerámico y vegetación. - Estrato II, contiene el derrumbe del muro, sedimento eólico suelto de color beige y escaso material arqueológico. Su potencia es de aproximadamente $40 \mathrm{~cm}$. Al remover el sedimento y los escombros se pudo observar que los cimientos de la pared se conservaban perfectamente, tratándose de un recinto circular demarcado por un solo muro.

- Estrato III, se inicia a los $40 \mathrm{~cm}$ de profundidad, en este se registró la mayor concentración de hallazgos arqueológicos y manchas de tierra quemada. El sedimento es de color grisáceo de textura fina y compacta, con una potencia de $15 \mathrm{a} 20 \mathrm{~cm}$. Este fue identificado como el nivel de ocupación.

- Estrato IV, presenta el sedimento rojizo de la roca madre con abundante pedregullo, roca suelta y escaso material arqueológico. Su potencia varía entre 10 y $15 \mathrm{~cm}$ y se asienta sobre la roca madre de la lomada que tiene una pendiente leve de sur a norte. De este estrato proviene la muestra de carbón fechada LP 1798 que tiene un fechado de $1000 \pm 70$ A.P.(1 sigma 1024 - 1154 AD; 2 sigma 978 - 1223 AD).

Después de realizar las excavaciones se pudo identificar que el recinto es de forma circular casi perfecta, con diámetros de 4,16 m N-S y 4,19 m E-O. La pared fue levantada con piedras canteadas de forma prismática, de manera similar a lo registrado en el R1 y en la mayoría de 
los recintos circulares del sitio (Albeck 1997 y 1999), con la diferencia de que en el R3 estas se presentan muy erosionadas y friables. En el interior del recinto el muro se hallaba revestido con un grueso revoque compuesto por un sedimento fino de color amarillo.

En el cuadrante NO del muro se registró un acceso de aproximadamente $60 \mathrm{~cm}$ de ancho, el sedimento del piso de ocupación en este espacio era compacto y grisáceo.

Junto al acceso se ubica un deflector conformado por una pequeña pared de rocas planas clavadas de manera vertical y sostenida en la base por rocas más pequeñas. Casi en el centro del recinto se ubicó un fogón delimitado por piedras chatas y alargadas puestas verticalmente para contener los elementos de combustión. Aproximadamente a un metro de la entrada se encontró el enterratorio directo de un individuo perinato (Figura 2).

\section{Análisis de materiales provenientes del Estrato III - Piso de Ocupación. Procesos de Formación.}

Mediante el registro de la procedencia de los fragmentos remontados (Nelson 1985; Albeck et al. 1995), en el R3 se diferencian dos niveles en el piso de ocupación (Estrato III): superior e inferior.

Se remontaron dos grandes vasijas $\left(\mathrm{N}^{\circ} 1 \mathrm{y}\right.$ 2) que presentaron estados de remontajes $1,2 \mathrm{y}$ 3 , todos sus fragmentos provenían del nivel superior del piso de ocupación. Dicho nivel comprende el tope del estrato III, es decir el sedimento sobre el que asienta el derrumbe del

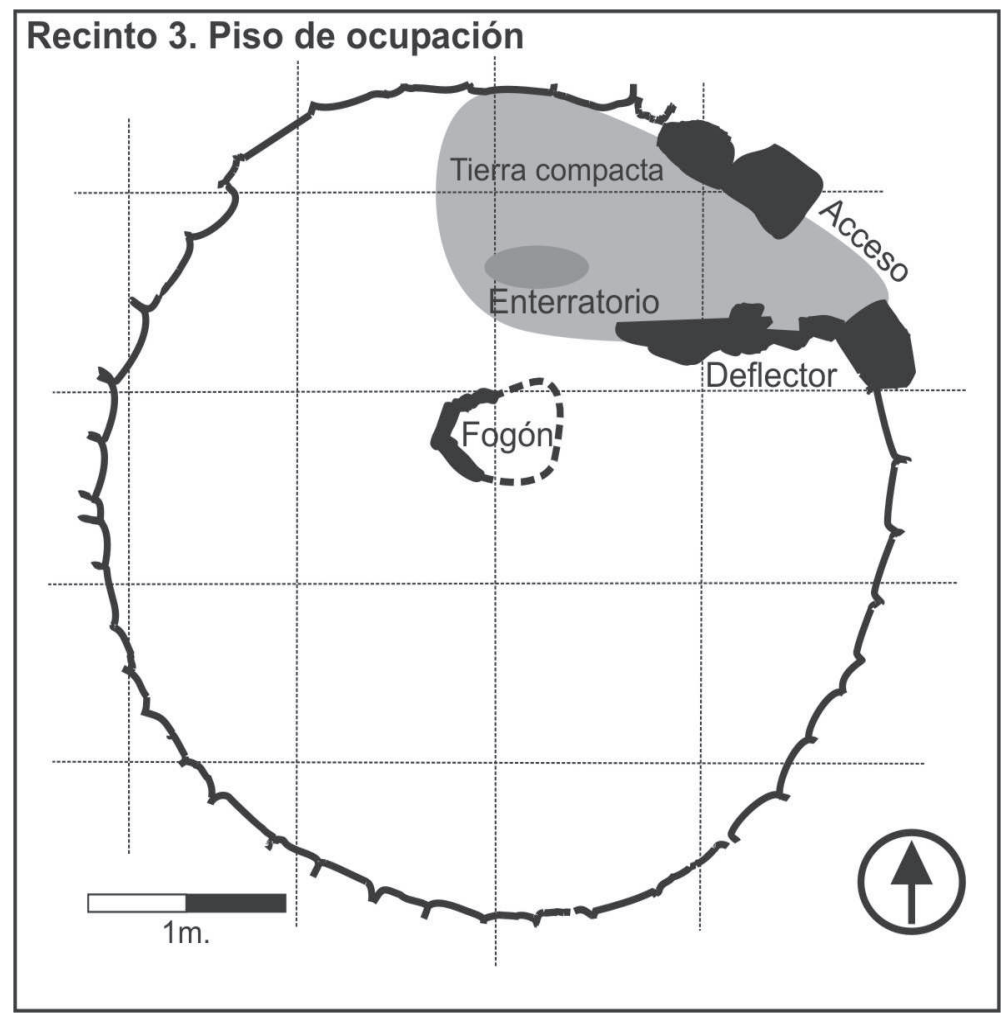

Figura 2: Recinto 3. Piso de Ocupación. 
muro y es posible plantearlo como el piso correspondiente al momento de abandono del recinto.

Con los fragmentos provenientes del nivel inferior del piso de ocupación se pudieron reconocer estados de remontaje 4, 5 y 6 , dichos fragmentos fueron clasificados a nivel macroscópico por sus atributos morfológicos y por similitud de tratamiento de superficie y pasta. Este nivel correspondería al piso de ocupación durante el uso de la vivienda.

Por otra parte, a partir del análisis de las estratigrafías de los remontajes y en base a observaciones en terreno se puede plantear que el nivel de ocupación no fue afectado por procesos postdepositacionales que alteraran la distribución vertical de los materiales.

Si embargo se registran movimientos postdepositacionales horizontales en el nivel superior del piso de ocupación. Las piezas remontadas 1 y 2 presentan el $80 \%$ del cuerpo, notándose que hay sectores que están ausentes del recinto. Inclusive el límite del cuerpo reconstruido no muestra evidencia de desgaste, por lo tanto las piezas no fueron reutilizadas luego de su fractura. Es factible plantear que estas piezas estaban enteras en el interior de la vivienda al momento de abandono y, por motivos diversos, partes de las vasijas presentan movimientos hacia el exterior de la misma.

\section{Análisis del material cerámico}

A continuación se describe el material cerámico recuperado de los dos niveles del piso de ocupación.

a) Material cerámico proveniente del nivel superior del piso de ocupación.

Dentro del conjunto de basura de facto, proveniente del nivel superior del piso de ocupación, se registran dos vasijas grandes remontadas ( $\mathrm{N}^{\circ} 1$ y 2 ), dos piezas enteras medianas ( $\mathrm{N}^{\circ} 3$ y 4 ), que se encontraban enterradas con su abertura a ras del nivel superior del piso, y una vasija pequeña ( No $^{\circ}$ ) (Figura 3 ).

Pieza №1: cántaro subovoide de contorno restringido inflexo con el diámetro máximo en el cuerpo superior y con asas horizontales en el hombro de la pieza, tiene $56 \mathrm{~cm}$ de altura y $42 \mathrm{~cm}$ de diámetro máximo, el cuello es corto y angosto con $23 \mathrm{~cm}$ de diámetro de abertura, mientras que la base tiene de $10 \mathrm{~cm}$ de diámetro, presenta un leve baño de pintura roja sobre la superficie externa y carece de huellas de uso.

Se remontó más de la mitad de la pieza faltando la base, se registran varios fragmentos con estados de remontaje 2 y 3 , los cuales, debido a la fragilidad de la pasta no han podido remontarse.

Pieza № 2: cántaro oval con cuello levemente convexo y asas en la mitad del cuerpo. La altura total debió ser un poco mayor a $54 \mathrm{~cm}$, el diámetro máximo es de $48 \mathrm{~cm}$ y la boca presenta $26 \mathrm{~cm}$ de diámetro. Tiene dos grandes asas ubicadas en el punto de diámetro máximo en el cuerpo superior. Estuvo pintada con un leve baño rojo y motivos de líneas negras oblicuas paralelas, pero la decoración se encuentra borrada. No se registran huellas de uso. 


\section{a)Piezas remontadas y enteras.}

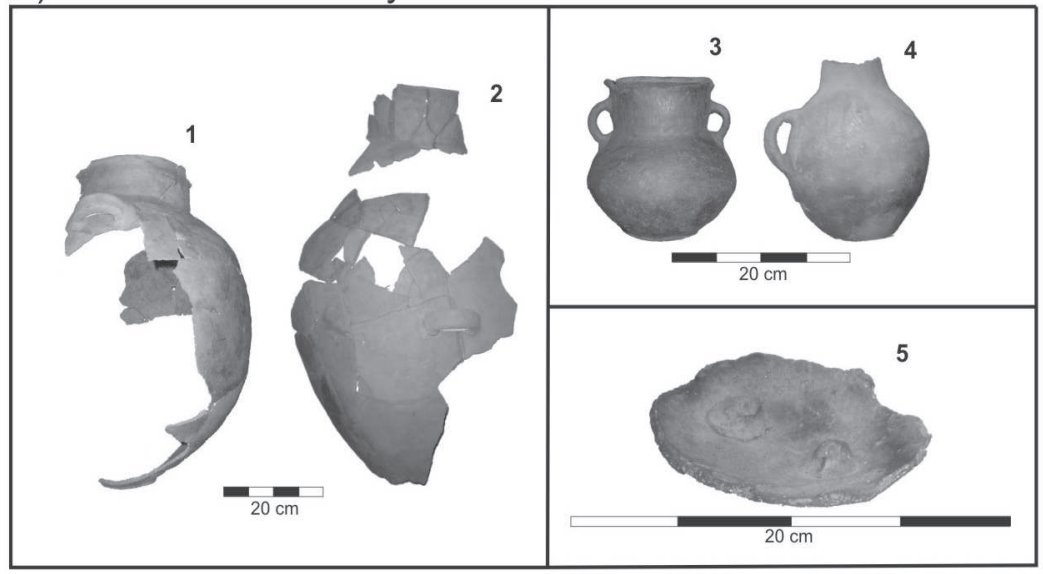

B)Comparación de los tipos de piezas provenientes de los dos niveles del piso de ocupación

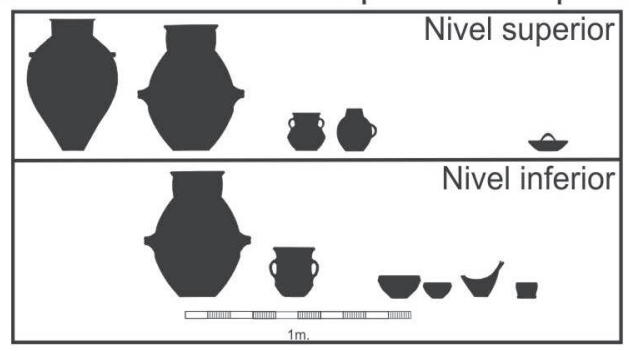

Figura 3: A- Piezas enteras del piso de ocupación. B-Formas reconocidas en el piso de ocupación

Las piezas $\mathrm{N}^{\mathrm{o}} 1$ y 2 tienen una capacidad de más de 15 litros, son altas con bases pequeñas y presentan gruesas asas en el cuerpo superior, todo lo cual permite inclinarlas con facilidad. Los cuellos son angostos factibles de tapar para proteger el contenido. La ausencia de huellas de uso permite plantear que fueron piezas con baja movilidad y no estuvieron expuestas al fuego. Estas características permiten clasificarlas como piezas apropiadas para el almacenaje de grandes cantidades de líquidos o sólidos.

Pieza No3: cántaro de contorno compuesto con el diámetro máximo en el punto angular, marcando el límite entre el cuerpo superior y el inferior. Presenta un cuello recto con asas verticales que unen el cuello con el cuerpo superior. Tiene decoración negro sobre rojo pulido que presenta ciertas similitudes con el tipo de decoración del área chicha (Ávila 2006). Tiene $17 \mathrm{~cm}$ de altura y $12 \mathrm{~cm}$ de diámetro de boca, con una capacidad de 1,5 litros. La pieza se encuentra en muy buen estado de conservación, aunque se registran sectores erosionados por el uso en las superficies externa e interna de la base y en la superficie externa del cuerpo inferior.

Los atributos formales y las huellas de uso permiten plantear que la función inicial de la pieza implicó determinados movimientos como manipular sólidos o líquidos, la abertura de 
la boca permite el acceso cómodo al contenido y también es posible taparla. La ausencia de hollín indica que no fue utilizada para ser expuesta al fuego. Sin embargo, en algún punto de su vida útil fue enterrada, con la abertura a ras del piso.

Pieza $\mathrm{N}^{\circ} 4$ : Botella de cuerpo globular y asa horizontal, tiene $20 \mathrm{~cm}$ de alto y la abertura presenta 6,5 cm de diámetro, su capacidad es de 1,9 litros. La pieza está alterada por procesos posdepositacionales, la pasta tiene abundante mica, lo cual genera que las paredes se desgranen con facilidad. Se encuentran muy afectados el borde, parte del cuello y un costado de la pieza. Sus atributos morfológicos son apropiados para la manipulación de líquidos (una sola asa grande y cuello angosto).

A esta pieza se la encontró enterrada, situación por la cual dicha pieza pierde sus atributos para verter líquidos ${ }^{1}$ y no es apta para guardar elementos debido a lo angosto del cuello.

Pieza $N^{\circ}$ 5: Plato con un asa en cinta adherida en el interior, este tipo de pieza ha sido denominado "bowls de hilar" por Alfaro de Lanzone (1988:96) quien por analogía etnográfica ha planteado que consisten en herramientas para separar madejas de colores durante el proceso de tejido, esta pieza presenta los bordes fragmentados por el uso.

b) Material cerámico proveniente del nivel inferior del piso de ocupación.

En el nivel inferior del piso de ocupación se registraron 267 fragmentos cerámicos, aquellos con atributos morfológicos permitieron reconocer diversas formas específicas y otros fueron agrupados en formas generales:

Vasijas grandes: se identificaron formas con cuello inflexo y con cuello corto similares a las piezas $\mathrm{N}^{\circ} 1$ y 2 , además se incluyeron en este conjunto los fragmentos cuyas dimensiones y curvatura indicaban que pertenecieron a algún tipo de pieza grande cerrada.

Vasijas medianas: se identificaron formas cerradas con cuello y vasijas de boca ancha. Las vasijas cerradas presentan distintas variedades de cuellos evertidos, la mayoría tiene pasta con abundante mica. Los fragmentos de vasijas de boca ancha corresponden a cántaros subcilíndricos de contorno compuesto, con tratamiento rojo pulido y decoración de rectángulos reticulados. Este tipo de pieza fue identificado como característico de la cerámica de Agua Caliente de Rachaite por Ottonello (1973:42 y 50).

Vasijas pequeñas: se identificaron fragmentos de pucos, vasos chatos y una vasija zoomorfa. Los fragmentos de puco pertenecieron a piezas pequeñas, en su mayoría corresponden con pucos de interior negro pulido y, en menor cantidad, se registraron pucos ordinarios y de interior rojo pulido. También se reconocieron fragmentos de vasos chatos. Este tipo de piezas es conocido como vasos de hilandera y se asocian con actividades de tejido. Son pequeños vasos de paredes finas que suelen presentar oquedades en la superficie interna de la base, generadas por el movimiento del huso (Krapovickas: 1958-59). Se registró un fragmento de vasija zoomorfa, se trata de una pieza troncocónica con un apéndice modelado zoomorfo representando el cuello y la cabeza de un camélido. Este tipo de vasija es posible analogarla con la forma actual denominada "chuiayuro" (Menacho 2000:41; 2007:21), en la actualidad estas piezas se utilizan en actividades rituales vinculadas con la reproducción del ganado. 


\begin{tabular}{|c|c|c|c|}
\hline \multicolumn{2}{|c|}{$\begin{array}{c}\text { Tipos morfológicos reconocidos en nivel } \\
\text { inferior del piso de ocupación }\end{array}$} & $\begin{array}{l}\text { Número } \\
\text { mínimo }\end{array}$ & $\begin{array}{l}\text { Número } \\
\text { máximo }\end{array}$ \\
\hline \multicolumn{2}{|c|}{ Pucos } & 25 & 69 \\
\hline \multicolumn{2}{|l|}{ Vas os chato } & 3 & 6 \\
\hline \multicolumn{2}{|c|}{ Yurozoomorfo } & 1 & 1 \\
\hline \multicolumn{2}{|c|}{ Piezas medianas abiertas } & 2 & 3 \\
\hline \multicolumn{2}{|c|}{ Piezas medianas cerradas con cuello } & 5 & 8 \\
\hline \multicolumn{2}{|c|}{ Piezas grandes cerradas con cuello } & 13 & 81 \\
\hline \multicolumn{2}{|c|}{ Piezas cerradas no determinadas } & - & 89 \\
\hline \multirow[t]{2}{*}{ Alfareria Chicha } & Pucos & 2 & 4 \\
\hline & $\begin{array}{l}\text { Piezas medianas cerradas } \\
\text { con cuello }\end{array}$ & 2 & 6 \\
\hline \multicolumn{2}{|l|}{ Total } & 53 & 267 \\
\hline
\end{tabular}

Tabla Nº 1.Número Máximo de fragmentos y Número Mínimo de vasijas registradas en el nivel inferior del piso de ocupación del R3-Tu-1.

En la categoría de "piezas cerradas no determinadas" se agruparon los fragmentos de cuerpo que no podían asignarse a ninguna de estas categorías morfológicas específica. Por último se registraron fragmentos de vasijas provenientes de la región chicha, con pasta muy compacta y decoración color ante y rojo pulido; corresponden a fragmentos de pucos y de piezas cerradas de tamaño mediano.

En la tabla $\mathrm{N}^{\circ} 1$ se resumen los distintos tipos de piezas reconocidas en el nivel inferior del piso de ocupación del R-3, acompañado de los cálculos de número mínimo y máximo para cada tipo.

Es necesario subrayar que las piezas más representadas son los pucos y las piezas grandes cerradas con cuello.

Al comparar el tipo de piezas representada en el conjunto de basura de facto del nivel superior y la basura primaria proveniente del nivel inferior del piso de ocupación se puede observar que ambos conjuntos presentan diferencias (Tabla No2) (Figura 3.b).

Las piezas pequeñas se encuentran representadas con mayor variabilidad y cantidad en el nivel inferior, principalmente los pucos, estando ausentes en el nivel superior. El hecho de que los pucos no estén entre la basura de facto puede relacionarse con que son objetos de uso personal y fácil traslado durante su vida útil.

Por otra parte, la alta representación de este tipo de piezas de consumo individual en el nivel inferior puede considerarse como indicador de que la vivienda tuvo un período de ocupación prolongado (Menacho 2000:89; 2001: 137 y 141).

Las piezas medianas (abiertas y cerradas) y las piezas cerradas grandes para almacenaje se encuentran presentes en ambos niveles. En cambio los fragmentos de piezas de origen chicha solamente se registran en el nivel inferior del piso de ocupación. 


\begin{tabular}{|c|c|c|c|}
\hline \multicolumn{2}{|c|}{ Tipo morfológico } & \multirow{2}{*}{$\begin{array}{c}\begin{array}{c}\text { Nivel } \\
\text { Superior }\end{array} \\
0\end{array}$} & \multirow{2}{*}{$\begin{array}{c}\begin{array}{c}\text { Nivel } \\
\text { Inferior }\end{array} \\
X\end{array}$} \\
\hline Piezas Pequeñas & Yurozoomorfo & & \\
\hline & $P_{11 c o s}$ & 0 & $x$ \\
\hline & Vasos chatos & 0 & $\mathrm{x}$ \\
\hline & Plato c on asa & $X$ & 0 \\
\hline \multirow{2}{*}{$\begin{array}{c}\text { Piezas } \\
\text { Medianas }\end{array}$} & Abiertas & $x$ & $x$ \\
\hline & $\begin{array}{l}\text { Cerradas c on cuello } \\
\text { (cantaros y botellas) }\end{array}$ & $\mathrm{X}$ & $\mathrm{X}$ \\
\hline Piezas Grandes & Cerradas c on cuello & X & X \\
\hline \multirow{3}{*}{$\begin{array}{l}\text { No } \\
\text { Determinadas } \\
\text { Alfareria Chicha }\end{array}$} & Piezas cerradas & 0 & X \\
\hline & $P_{11 c o s}$ & 0 & X \\
\hline & $\begin{array}{l}\text { Piezas medianas } \\
\text { cerrada con cuello }\end{array}$ & 0 & $\mathrm{x}$ \\
\hline
\end{tabular}

Tabla No 2. Comparación de los tipos y tamaños de piezas representadas en los dos niveles del piso de ocupación del R3-Tu-1.

En todo el conjunto cerámico del R3 no se encuentran representadas las etapas de manufactura cerámica, pero si se registraron elementos de las etapas de uso (cerámica con exposición al fuego, vasos de hilandera con huellas de uso) y mantenimiento (2 fragmentos con perforaciones para reparar) (Schiffer 1979).

Es necesario remarcar también que los fragmentos de piezas con exposición al fuego se registran únicamente el nivel inferior del piso de ocupación.

\section{Reconstrucción de áreas de actividad}

\section{Área de Almacenaje}

La presencia de vasijas con una capacidad mayor a 15 litros es considerada como un indicador de almacenaje en grandes volúmenes en el interior del recinto, las vasijas de este tipo que remontaban procedían del nivel superior del piso de ocupación (piezas $N^{\circ} 1$ y 2). Es posible considerar también que hayan sido guardadas vacías dentro del recinto, aunque el hecho de que se registren abundantes fragmentos de piezas grandes en el nivel inferior del piso de ocupación, indicaría que vasijas similares fueron utilizadas dentro del recinto durante su ocupación.

La dispersión de los fragmentos de las piezas $N^{\circ} 1$ y 2 se concentra en el sector $\mathrm{SW}$, del recinto cerca de la pared, ante lo cual es posible considerar esta zona como su última ubicación antes del abandono del recinto. Al estar junto al muro no habrían obstruido el paso dentro del recinto, permitiendo la circulación en el espacio central alrededor del fogón.

La pieza No3 se encontraba enterrada en el cuadrante NW junto al muro y pudo utilizarse para almacenar elementos en volúmenes menores o quizás pudo haber tenido funciones no utilitarias; hasta el momento faltan análisis que nos brinden elementos diagnósticos al respecto. 
Área de procesamiento y consumo de alimentos

Existen variados elementos que fueron planteados como indicadores de actividades de procesamiento de alimentos. En primer lugar se considera la presencia de un fogón delimitado por piedras clavadas, caracterizado como elemento semifijo (Rapoport 1990:13). En cuanto a otros materiales, se registraron artefactos líticos como manos de moler y piedras planas desgastadas por el uso (el material lítico está en proceso de análisis) y astillas de restos óseos, algunas de las cuales se encuentran quemadas. El conjunto del material cerámico que se vinculó con la actividad de procesamiento de alimento son los restos de vasijas medianas (cuyas dimensiones facilitan la manipulación de líquidos y sólidos) y los fragmentos con indicios de exposición al fuego.

Los elementos que se consideraron diagnósticos de actividades de consumo de alimentos fueron los fragmentos de piezas pequeñas abiertas (pucos). Las áreas de dispersión demarcadas por estos elementos comprenden los cuadrantes NE, NW y SW.

\section{Descanso}

Como se mencionó anteriormente, la mayor cantidad de hallazgos se dispersaba en los sectores NE, NW y SW, presentando muy baja densidad en el SE, detrás del deflector. Dicho sector debió constituir el espacio con mejores condiciones para el resguardo, ya que cuenta con la protección del deflector contra el viento y se halla cerca del fogón. Un espacio de la misma naturaleza y ubicación relativa fue registrado en el Recinto 1.

Área de Inhumación

En el cuadrante NE, en el espacio adyacente al acceso del recinto, se encontraba un entierro directo sin elementos que lo delimitaran, ni indicios en superficie. Los restos óseos pertenecían a un individuo perinato (Mendonça $\mathrm{ms}$ ) y no se registraron elemento que pudieran considerarse como ajuar.

\section{Otras actividades}

Tejido

Algunos elementos cerámicos se pueden vincular con la actividad textil, específicamente con las etapas de hilado y tejido: los fragmentos de vasos de hilandera en el nivel inferior del piso de ocupación y el plato con asa en su interior (pieza No5) en el nivel superior. Se ha observado que la población local realiza las tareas de hilado en cualquier momento y lugar, dentro y fuera de las viviendas, ya que los elementos necesarios (vellón, huso y apoyo) son elementos de fácil transporte.

En el R3 no se puede delimitar un área específica para esta actividad que incluso debió desarrollarse fuera del recinto aunque, en el caso de la pieza №5, se podía considerar que había estado depositada o guardada dentro del recinto. Pero la presencia de fragmentos de vasos de hilandera en el nivel inferior remarca que dicha actividad fue realizada por los habitantes de la vivienda. 


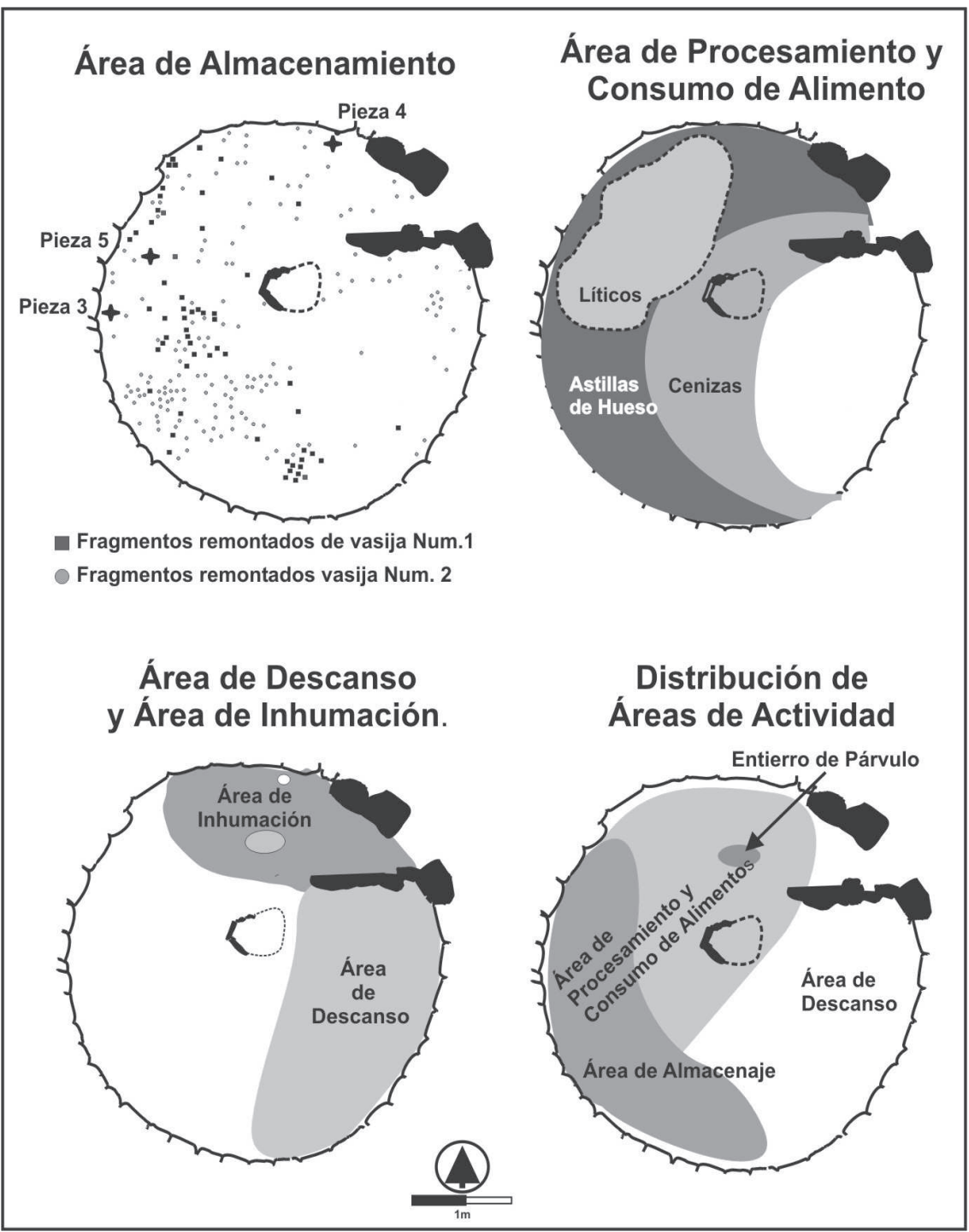

Figura 4: Areas de Actividad Recinto R3.

\section{Actividades Rituales}

Asociado al acceso se ubicaba una de las vasijas enterradas, la pieza $\mathrm{N}^{\circ} 4$. Como se planteó anteriormente esta pieza con cuello angosto no es operativa para actividades de almacenaje, ante lo cual es posible pensar que estuviese vinculada con actividades rituales, tal vez relacionada con la inhumación.

Por último, un elemento que podría vincularse con actividades rituales relacionadas al ganado (en base a analogías etnográficas) es la presencia del fragmento de la vasija zoomorfa que se mencionó anteriormente.

Sintetizando, las áreas de actividad reconocidas dentro del recinto fueron: área de almacenaje, área de procesamiento y consumo de alimentos, área de descanso y área de 
inhumación. Se identificaron además actividades que no tienen una representación espacial específica dentro de la vivienda, como las actividades de hilado y posiblemente actividades rituales (figura 4 ).

\section{Reflexiones finales}

En la introducción se planteaba la importancia que cobran los estudios de áreas de actividad para la comparación entre recintos de un mismo sitio en busca de indicios sobre diferencias sociales o marcas de identidad social. En este caso el análisis de actividades y de la arquitectura en el R1 y en el R3 permite problematizar sobre indicadores de organización social del trabajo en la población.

Las actividades productivas en cada recinto no son idénticas, en el R1 se identificaron áreas de manufactura de cerámica y de piezas líticas. Dichas actividades están ausentes en el R3 donde se encuentra representada parte del proceso de producción textil. Aunque se debe considerar también el hecho de que aún no está definido el equivalente arquitectónico del grupo corresidencial mínimo y por lo tanto las actividades en los recintos pudieron realizarse en espacios adyacentes que aún no se han excavado.

Por otra parte, en los dos recintos excavados, como en la mayoría del sitio, se levantaron las paredes con rocas talladas, canteadas como un prisma rectangular, dando lugar a que los muros de los recintos circulares fueran muy homogéneos. Estas características uniformes en las estructuras circulares llevan a preguntarse sobre la posibilidad de la existencia de un grupo de especialistas lapidarios. También al momento de construir una vivienda debió requerirse la colaboración de varios miembros de la comunidad.

Otro aspecto sobre la organización social que puede discutirse a partir del análisis comparativo entre los dos recintos consiste en el almacenaje. Las diferencias en la cantidad de piezas de almacenaje registradas en ambos recintos es notoria: seis en el R1 y dos en el R3. Las mismas fueron registradas en el nivel superior del piso de ocupación. Estas diferencias podrían indicar la presencia de grupos con distinto grado de control y acceso a los recursos almacenados, lo cual se vincula con diferencias jerárquicas dentro de la población. Sin embargo se debe considerar la posibilidad de que estemos ante diferentes procesos de abandono. En el R3 se puede sostener que las grandes piezas de almacenaje fueron utilizadas durante la ocupación de la estructura, sin embargo en el R1 aun no se han analizado los materiales del nivel inferior del piso de ocupación, esto último no permite por el momento establecer si el interior del recinto conformaba un espacio para guardar las piezas o si éstas se utilizaban para almacenaje.

A partir del estudio de la organización del espacio en el interior de los recintos circulares, se observa no obstante que, entre el R1 y el R3, existen pautas compartidas.

A pesar de que las entradas a los recintos no tienen la misma orientación (el acceso del R1 se orienta hacia el S, mientras que en el R3 se halla orientado hacia el NE), la organización interior sigue un patrón similar, al encontrarse estructuradas y condicionadas por dos elementos semifijos: un fogón en el centro del recinto y el deflector situado frente a la puerta. 
Las áreas delimitadas para procesamiento y consumo de alimentos en ambos recintos estaban cerca del fogón y en los lugares donde recibían más luz. En tanto el área vacía, denominada de "descanso", se encontraba entre el fogón y el deflector en ambos recintos.

Además es notorio destacar que en estos ambos recinto se encontraron entierros directos de perinatos en el espacio cercano al acceso. Hasta el momento no se puede identificar si los enterratorios se realizaron mientras que la vivienda se encontraba en uso o con posterioridad a su abandono, como ha sido registrado en la Quebrada de Humahuaca (Tarragó 1992; Taboada 2003).

Otro elemento que se encuentra presente en la dos viviendas, con la particularidad de estar representados en bajas proporciones son los fragmentos de vasijas zoomorfas.

Las actividades mencionadas en este artículo seguramente no son las únicas que se realizaron en la antigua vivienda. Por observación directa en el poblado actual de Casabindo y dadas las condiciones climáticas de la zona se puede asegurar que la mayoría de las actividades domésticas se realizaron a la intemperie, en los patios de las viviendas. Los espacios interiores son ocupados durante las horas mas frías, por las tardes y noches; mientras que durante el día la vivienda es oscura y fría comparada con el exterior.

En futuras excavaciones se espera trabajar espacios abiertos que pudieron funcionar como patios, para abarcar un espectro más amplio de las distintas actividades que desarrollaban los antiguos habitantes del Pueblo Viejo de Tucute.

\section{Notas}

${ }^{1}$ El sedimento encontrado en el interior de las piezas 3 y 4 fue separado para futuros análisis

\section{Bibliografía citada}

Albeck, M.E.

1997. Áreas de Actividad Doméstica en Pueblo Viejo de Tucute. Estudios Atacameños 12. Universidad Católica del Norte. Chile

1999. El recinto R-1 de Pueblo Viejo de Tucute: Sorcuyo Revisitado. Chungará 130:143-159. Arica. Chile.

2007. El Intermedio Tardío: interacciones económicas y políticas en la Puna de Jujuy. En V.I.Williams, B.N.Ventura, A.B.Callegari y H.D. Yacobaccio. (Eds.) Sociedades Precolombinas Surandinas. Temporalidad, Interacción y Dinámica cultural del NOA en el ámbito de los Andes Centro-Sur:125-146. Bs. As.

2009. Pueblo Viejo de Tucute. Una sociedad interpretada a través de la construcción del espacio. El hábitat prehispánico: Arqueología de la arquitectura y de la construcción del espacio organizado. (ed. Albeck, Korstanje y Scattolin). (En prensa)

Albeck M.E; Dip S.y Zaburlín M.A.

1998 El patrón arquitectónico de Pueblo Viejo de Tucute. En “Los Desarrollos Locales y sus Territorios".: 223-244. (Compiladora María Beatriz Cremonte.) UNJu.

Albeck, M., H. Mamaní. y M. Zaburlín.

1995. La cerámica del R-1 de Pueblo Viejo de Tucute. Primera Parte: Función y Dispersión de Vasijas. Cuadernos 5:205-220. FHyCS-UNJu. Jujuy. 
Albeck, M. y M. Zaburlín

2007. Lo público y lo privado en Pueblo Viejo de Tucute. Procesos sociales prehispánicos en el Sur Andino. La vivienda, la comunidad, el territorio. (Comp. A.E. Nielsen y otros). pp. 163-181. Editorial Brujas

2008. Aportes a la cronología de los asentamientos agropastoriles de la Puna de Jujuy. Revista Relaciones de la Sociedad Argentina de Antropología XXXIII pp. 155-186.Buenos Aires.

Alfaro de Lanzone, L.

1988. Excavación de la Cuenca del Río Doncellas. Reconstrucción de una Cultura olvidada en la Puna Jujeña. Jujuy

Avila, F.

2006. Un mundo morado sobre ante. Tesis de Licenciatura. Facultad de Filosofia y Letras. Carrera de Antropologia. UBA. Manuscrito

Binford, L.

1981. Behavioral Archaeology and the "Pompeii Premise". Journal of Anthropological Research. 37 (3):1925-208. Traducción: Mirta Bonín.

Blanton, R.

1994. Houses and households. A comparative study. Plenum Press. New York and London.

Bollong, C. A.

1994. Analysis of Site Stratigraphy and Formation Processes Using Patterns of Pottery Sherd Dispersion. Journal of Field Archaeology Vol.2:15-28

Casanova, E.

1938. Investigaciones Arqueológicas en Sorcuyo, Puna de Jujuy. Anales del Museo Argentino de Ciencias Naturales 80(39):423-462. Buenos Aires.

Krapovickas, $\mathrm{P}$.

1958-59 Arqueología de la Puna Argentina. Anales de Arqueología y Etnología 14-15:53113.Universidad Nacional de Cuyo- Facultad de Filosofía y Letras.

Menacho, K.

2000. Trayectoria de Vida de Vasijas Cerámicas y Modo de Vida Pastoril. Tesis de Licenciatura. FHyCS. UNJu.

2001. Etnoarqueología de trayectorias de vida de vasijas cerámicas. Relaciones de la Sociedad Argentina de Antropología XXVI: 120-143 Bs. As.

2007. Etnoarqueología y estudio sobre funcionalidad cerámica: aportes a partir de un caso de estudio. Intersecciones en Antropología 8: 15-26. FCS-UNCPBA.

Mendonça, $\mathrm{O}$.

2006. Informe sobre análisis de restos óseos humanos provenientes de Pueblo Viejo de Tucute.

Nelson, B.A.

1985. Reconstructing Ceramic Vessels and their systemic contexts. Decoding Prehistoric Ceramics. Editado por B.A. Nelson. Carbondales: Southern Illinois University Press. 
Rapoport, A.

1990 Systems of activities and systems of settings. Domestic architecture and the use of space. An interdisciplinary cross - cultural study.: 9-20. Ed. Susan Kent. Cambridge University Press.

Ottonello de García Reinoso, M.

1973 Instalación, economía y cambio cultural en el sitio tardío de Agua Caliente de Rachaite. En publicaciones N ${ }^{o}$ 1:24-68. Dirección de Antropología e Historia. S.S. de Jujuy.

Sheppard, A.

1965. Ceramics for the archaeologists. Carnegie Institution, Washington D.C.

Schiffer, M.B.

1979. Contexto arqueológico y contexto sistémico. Boletín de Antropología Americana 2: 81-93 Original publicado en American Antiquity 37 (2) 1972.

1987. Formation Processes of the Archaeological Record. University of New Mexico Press. Albuquerque.

1988 ¿Existe una "Premisa Pompeya" en arqueología? Boletín de Antropología Americana18.:531. Original publicado en Journal of Anthropological. Research 41 (1) 1985.

Taboada, C.

2003. Arquitectura y sociedad en la Quebrada de Humahuaca prehispánica. Tesis Doctoral. Facultad de Ciencias Naturales e Instituto Miguel Lillo. Universidad Nacional de Tucumán.

Tarragó, M.

1992. Áreas de actividad y formación del sitio de Tilcara. Cuadernos 3: 64-74. FHyCS-UNJu.

Wandsnider, L.A.

1996. Describing and comparing archaeological spatial structures. Journal of archaeological Method and Theory. Vol 3 No.4 Plenum Press: 319-384. 